\title{
A MAP Estimator for Simultaneous Superresolution and Detector Nonunifomity Correction
}

\author{
Russell C. Hardie ${ }^{1}$ and Douglas R. Droege ${ }^{2}$ \\ ${ }^{1}$ Department of Electrical and Computer Engineering, University of Dayton, 300 College Park, Dayton, OH 45469-0226, USA \\ ${ }^{2}$ L-3 Communications Cincinnati Electronics, 7500 Innovation Way, Mason, OH 45040, USA
}

Received 31 August 2006; Accepted 9 April 2007

Recommended by Richard R. Schultz

During digital video acquisition, imagery may be degraded by a number of phenomena including undersampling, blur, and noise. Many systems, particularly those containing infrared focal plane array (FPA) sensors, are also subject to detector nonuniformity. Nonuniformity, or fixed pattern noise, results from nonuniform responsivity of the photodetectors that make up the FPA. Here we propose a maximum a posteriori (MAP) estimation framework for simultaneously addressing undersampling, linear blur, additive noise, and bias nonuniformity. In particular, we jointly estimate a superresolution (SR) image and detector bias nonuniformity parameters from a sequence of observed frames. This algorithm can be applied to video in a variety of ways including using a moving temporal window of frames to process successive groups of frames. By combining SR and nonuniformity correction (NUC) in this fashion, we demonstrate that superior results are possible compared with the more conventional approach of performing scene-based NUC followed by independent SR. The proposed MAP algorithm can be applied with or without SR, depending on the application and computational resources available. Even without SR, we believe that the proposed algorithm represents a novel and promising scene-based NUC technique. We present a number of experimental results to demonstrate the efficacy of the proposed algorithm. These include simulated imagery for quantitative analysis and real infrared video for qualitative analysis.

Copyright (c) 2007 R. C. Hardie and D. R. Droege. This is an open access article distributed under the Creative Commons Attribution License, which permits unrestricted use, distribution, and reproduction in any medium, provided the original work is properly cited.

\section{INTRODUCTION}

During digital video acquisition, imagery may be degraded by a number of phenomena including undersampling, blur, and noise. Many systems, particularly those containing infrared focal plane array (FPA) sensors, are also subject to detector nonuniformity [1-4]. Nonuniformity, or fixed pattern noise, results from nonuniform responsivity of the photodetectors that make up the FPA. This nonuniformity tends to drift over time, precluding a simple one-time factory correction from completely eradicating the problem. Traditional methods of reducing fixed pattern noise, such as correlated double sampling [5], are often ineffective because the processing technology and operating temperatures of infrared sensor materials result in the dominance of different sources of nonuniformity. Periodic calibration techniques can be employed to address the problem in the field. These, however, require halting normal operation while the imager is aimed at calibration targets. Furthermore, these methods may only be effective for a scene with a dynamic range close to that of the calibration targets. Many scene-based techniques have been proposed to perform nonuniformity correction (NUC) using only the available scene imagery (without calibration targets).

Some of the first scene-based NUC techniques were based on the assumption that the statistics of each detector output should be the same over a sufficient number of frames as long as there is motion in the scene. In [6-9], offset and gain correction coefficients are estimated by assuming that the temporal mean and variance of each detector are identical over time. Both a temporal highpass filtering approach that forces the mean of each detector to zero and a leastmean squares technique that forces the output of a pixel to be similar to its neighbors are presented in [10-12]. By exploiting a local constant statistics assumption, the technique presented in [13] treats the nonuniformity at the detector level separately from the nonuniformity in the readout electronics. Another approach is based on the assumption that the output of each detector should exhibit a constant range of values [14]. A Kalman filter-based approach 
that exploits the constant range assumption has been proposed in [15]. A nonlinear filter-based method is described in [16]. As a group, these methods are often referred to as constant statistics techniques. Constant statistics techniques work well when motion in a relatively large number of frames distributes diverse scene intensities across the FPA.

Another set of proposed scene-based NUC techniques utilizes motion estimation or specific knowledge of the relative motion between the scene and the FPA [17-23]. A motion-compensated temporal average approach is presented in [19]. Algebraic scene-based NUC techniques are developed in [20-22]. A regularized least-squares method, closely related to this work, is presented in [23]. These motion-compensated techniques are generally able to operate successfully with fewer frames than constant statistics techniques. Note that many motion-compensated techniques utilize interpolation to treat subpixel motion. If the observed imagery is undersampled, the ability to perform accurate interpolation is compromised, and these NUC techniques can be adversely affected.

When aliasing from undersampling is the primary form of degradation, a variety of superresolution (SR) algorithms can be employed to exploit motion in digital video frames. A good survey of the field can be found in [24, 25]. Statistical SR estimation methods derived using a Bayesian framework, similar to that used here, include [26-30]. When significant levels of both nonuniformity and aliasing are present, most approaches treat the nonuniformity and undersampling separately. In particular, some type of calibration or scene-based NUC is employed initially. This is followed by applying an SR algorithm to the corrected imager [31,32]. One pioneering paper developed a maximum-likelihood estimator to jointly estimate a high-resolution (HR) image, shift parameters, and nonuniformity parameters [33].

Here we combine scene-based NUC with SR using a maximum a posteriori (MAP) estimation framework to jointly estimate an SR image and detector nonuniformity parameters from a sequence of observed frames (MAP SR-NUC algorithm). We use Gaussian priors for the HR image, biases, and noise. We employ a gradient descent optimization and estimate the motion parameters prior to the MAP algorithm. Here we focus on translational and rotational motion. The joint MAP SR-NUC algorithm can be applied to video in a variety of ways including processing successive groups of frames spanned by a moving temporal window of frames. By combining SR and NUC in this fashion, we demonstrate that superior results are possible compared with the more conventional approach of performing scene-based NUC followed by independent SR. This is because access to an SR image can make interpolation more accurate, leading to improved nonuniformity parameter estimation. Similarly, HR image estimation requires accurate knowledge of the detector nonuniformity parameters. The proposed MAP algorithm can be applied with or without SR, depending on the application and computational resources available. Even without SR, we believe that the proposed algorithm represents a novel and promising scene-based NUC technique (MAP NUC algorithm).

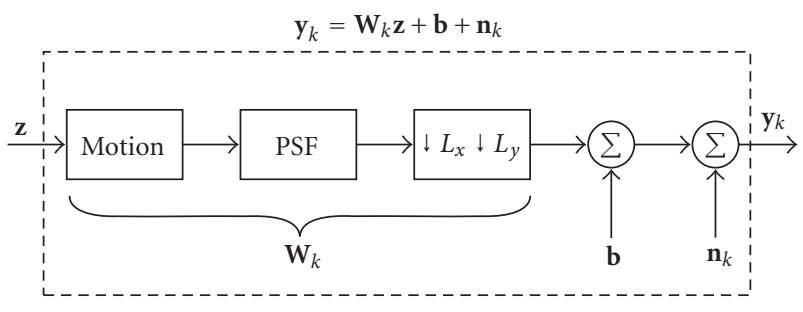

FIGURE 1: Observation model for simultaneous image superresolution and nonuniformity correction.

The rest of this paper is organized as follows. In Section 2, we present the observation model. The joint MAP estimator and corresponding optimization are presented in Section 3. Experimental results are presented in Section 4 to demonstrate the efficacy of the proposed algorithm. These include results produced using simulated imagery for quantitative analysis and real infrared video for qualitative analysis. Conclusions are presented in Section 5.

\section{OBSERVATION MODEL}

Figure 1 illustrates the observation model that relates a set of observed low-resolution (LR) frames with a corresponding desired HR image. Sampling the scene at or above the Nyquist rate gives rise to the desired HR image, denoted using lexicographical notation as an $N \times 1$ vector $\mathbf{z}$. Next, a geometric transformation is applied to model the relative motion between the camera and the scene. Here we consider rigid translational and rotational motion. This requires only three motion parameters per frame and is a reasonably good model for video of static scenes imaged at long range from a nonstationary platform. We next incorporate the point spread function (PSF) of the imaging system using a 2D linear convolution operation. The PSF can be modified to include other degradations as well. In the model, the image is then downsampled by factors of $L_{x}$ and $L_{y}$ in the horizontal and vertical directions, respectively.

We now introduce the nonuniformity by adding an $M \times 1$ array of biases, $\mathbf{b}$, where $M=N /\left(L_{x} L_{y}\right)$. Detector nonuniformity is frequently modeled using a gain parameter and bias parameter for each detector, allowing for a linear correction. However, in many systems, the nonuniformity in the gain term tends to be less variable and good results can be obtained from a bias-only correction. Since a model containing only biases simplifies the resulting algorithms and provides good results on the imagery tested here, we focus here on a bias-only nonuniformity model. Finally, an $M \times 1$ Gaussian noise vector $\mathbf{n}_{k}$ is added. This forms the $k$ th observed frame represented by an $M \times 1$ vector $\mathbf{y}_{k}$. Let us assume that we have observed $P$ frames, $\mathbf{y}_{1}, \mathbf{y}_{2}, \ldots, \mathbf{y}_{P}$. The complete observation model can be expressed as

$$
\mathbf{y}_{k}=\mathbf{W}_{k} \mathbf{z}+\mathbf{b}+\mathbf{n}_{k},
$$

for $k=1,2, \ldots, P$, where $\mathbf{W}_{k}$ is an $M \times N$ matrix that implements the motion model for the $k$ th frame, the system PSF 
blur, and the subsampling shown in Figure 1. Note that this model can accommodate downsampling (i.e., $L_{x}, L_{y}>1$ ) for SR or can perform NUC only for $L_{x}=L_{y}=1$. Also note that the operation $\mathbf{W}_{k} \mathbf{z}$ implements subpixel motion for any $L_{x}$ and $L_{y}$ by performing bilinear interpolation.

We model the additive noise as a zero-mean Gaussian random vector with the following multivariate PDF:

$$
\operatorname{Pr}\left(\mathbf{n}_{k}\right)=\frac{1}{(2 \pi)^{M / 2} \sigma_{n}^{M}} \exp \left\{-\frac{1}{2 \sigma_{n}^{2}} \mathbf{n}_{k}^{T} \mathbf{n}_{k}\right\}
$$

for $k=1,2, \ldots, P$, where $\sigma_{n}^{2}$ is the noise variance. We also assume that these random vectors are independent from frame to frame (temporal noise).

We model the biases (fixed pattern noise) as a zero-mean Gaussian random vector with the following PDF:

$$
\operatorname{Pr}(\mathbf{b})=\frac{1}{(2 \pi)^{M / 2} \sigma_{b}^{M}} \exp \left\{-\frac{1}{2 \sigma_{b}^{2}} \mathbf{b}^{T} \mathbf{b}\right\}
$$

where $\sigma_{b}^{2}$ is the variance of the bias parameters. This Gaussian model is chosen for analytical convenience but has been shown to produce useful results.

We model the HR image using a Gaussian PDF given by

$$
\operatorname{Pr}(\mathbf{z})=\frac{1}{(2 \pi)^{N / 2}\left|C_{z}\right|^{1 / 2}} \exp \left\{-\frac{1}{2} \mathbf{z}^{T} C_{z}^{-1} \mathbf{z}\right\},
$$

where $C_{z}$ is the $N \times N$ covariance matrix. The exponential term in (4) can be factored into a sum of products yielding

$$
\operatorname{Pr}(\mathbf{z})=\frac{1}{(2 \pi)^{N / 2}\left|C_{z}\right|^{1 / 2}} \exp \left\{-\frac{1}{2 \sigma_{z}^{2}} \sum_{i=1}^{N} \mathbf{z}^{T} \mathbf{d}_{i} \mathbf{d}_{i}^{T} \mathbf{z}\right\},
$$

where $\mathbf{d}_{i}=\left[d_{i, 1}, d_{i, 2}, \ldots, d_{i, N}\right]^{T}$ is a coefficient vector. Thus, the prior can be rewritten as

$$
\operatorname{Pr}(\mathbf{z})=\frac{1}{(2 \pi)^{N / 2}\left|C_{z}\right|^{1 / 2}} \exp \left\{-\frac{1}{2 \sigma_{z}^{2}} \sum_{i=1}^{N}\left(\sum_{j=1}^{N} d_{i, j} z_{j}\right)^{2}\right\} .
$$

The coefficient vectors $\mathbf{d}_{i}$ for $i=1,2, \ldots, N$ are selected to provide a higher probability for smooth random fields. Here we have selected the following values for the coefficient vectors:

$$
d_{i, j}= \begin{cases}1 & \text { for } i=j \\ -\frac{1}{4} & \text { for } j: z_{j} \text { is a cardinal neighbor of } z_{i} .\end{cases}
$$

This model implies that every pixel value in the desired image can be modeled as the average of its four cardinal neighbors plus a Gaussian random variable of variance $\sigma_{z}^{2}$. Note that the prior in (6) can also be viewed as a Gibbs distribution where the exponential term is a sum of clique potential functions [34] derived from a third-order neighborhood system $[35,36]$.

\section{JOINT SUPERRESOLUTION AND NONUNIFORMITY CORRECTION}

Given that we observe $P$ frames, denoted by $\mathbf{y}=$ $\left[\mathbf{y}_{1}^{T}, \mathbf{y}_{2}^{T}, \ldots, \mathbf{y}_{P}^{T}\right]^{T}$, we wish to jointly estimate the HR image $\mathbf{z}$ and the nonuniformity parameters $\mathbf{b}$. In Section 4 , we will demonstrate that it is advantageous to estimate these simultaneously versus independently.

\subsection{MAP estimation}

The joint MAP estimation is given by

$$
\hat{\mathbf{z}}, \widehat{\mathbf{b}}=\underset{\mathbf{z}, \mathbf{b}}{\arg \max } \operatorname{Pr}(\mathbf{z}, \mathbf{b} \mid \mathbf{y}) .
$$

Using Bayes rule, this can be equivalently be expressed as

$$
\widehat{\mathbf{z}}, \widehat{\mathbf{b}}=\underset{\mathbf{z}, \mathbf{b}}{\arg \max } \frac{\operatorname{Pr}(\mathbf{y} \mid \mathbf{z}, \mathbf{b}) \operatorname{Pr}(\mathbf{z}, \mathbf{b})}{\operatorname{Pr}(\mathbf{y})} .
$$

Assuming that the biases and the HR image are independent, and noting that the denominator in (9) is not a function of $\mathbf{z}$ or $\mathbf{b}$, we obtain

$$
\widehat{\mathbf{z}}, \hat{\mathbf{b}}=\underset{\mathbf{z}, \mathbf{b}}{\arg \max } \operatorname{Pr}(\mathbf{y} \mid \mathbf{z}, \mathbf{b}) \operatorname{Pr}(\mathbf{z}) \operatorname{Pr}(\mathbf{b}) .
$$

We can express the MAP estimation in terms of a minimization of a cost function as follows:

$$
\widehat{\mathbf{z}}, \widehat{\mathbf{b}}=\underset{\mathbf{z}, \mathbf{b}}{\arg \min }\{L(\mathbf{z}, \mathbf{b})\},
$$

where

$$
L(\mathbf{z}, \mathbf{b})=-\log [\operatorname{Pr}(\mathbf{y} \mid \mathbf{z}, \mathbf{b})]-\log [\operatorname{Pr}(\mathbf{z})]-\log [\operatorname{Pr}(\mathbf{b})] .
$$

Note that when given $\mathbf{z}$ and $\mathbf{b}, \mathbf{y}_{k}$ is essentially the noise with the mean shifted to $\mathbf{W}_{k} \mathbf{z}+\mathbf{b}$. This gives rise to the following PDF:

$$
\begin{aligned}
\operatorname{Pr}(\mathbf{y} \mid \mathbf{z}, \mathbf{b}) \\
=\prod_{k=1}^{P} \frac{1}{(2 \pi)^{M / 2} \sigma_{n}^{M}} \\
\quad \times \exp \left\{-\frac{1}{2 \sigma_{n}^{2}}\left(\mathbf{y}_{k}-\mathbf{W}_{k} \mathbf{z}-\mathbf{b}\right)^{T}\left(\mathbf{y}_{k}-\mathbf{W}_{k} \mathbf{z}-\mathbf{b}\right)\right\}
\end{aligned}
$$

This can be expressed equivalently as follows:

$$
\begin{aligned}
\operatorname{Pr}(\mathbf{y} \mid \mathbf{z}, \mathbf{b}) \\
=\frac{1}{(2 \pi)^{\mathrm{PM} / 2} \sigma_{n}^{\mathrm{PM}}} \\
\\
\quad \times \exp \left\{-\sum_{k=1}^{P} \frac{1}{2 \sigma_{n}^{2}}\left(\mathbf{y}_{k}-\mathbf{W}_{k} \mathbf{z}-\mathbf{b}\right)^{T}\left(\mathbf{y}_{k}-\mathbf{W}_{k} \mathbf{z}-\mathbf{b}\right)\right\} .
\end{aligned}
$$




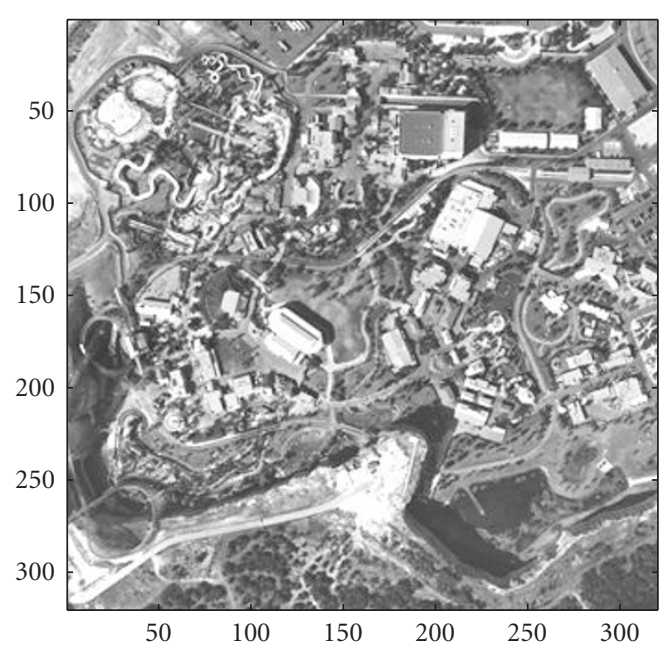

(a)

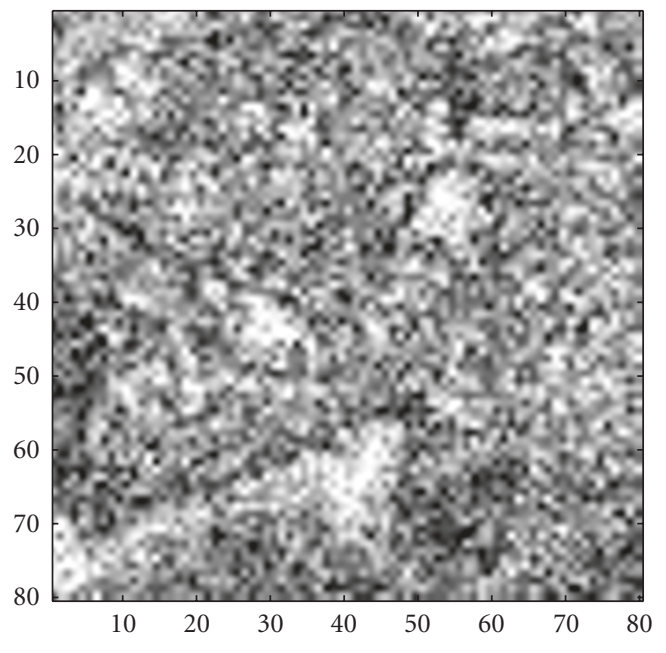

(c)

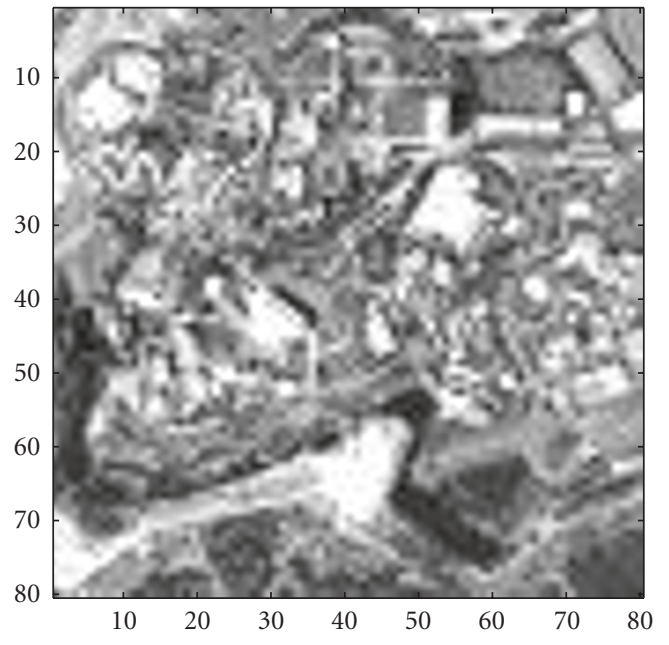

(b)

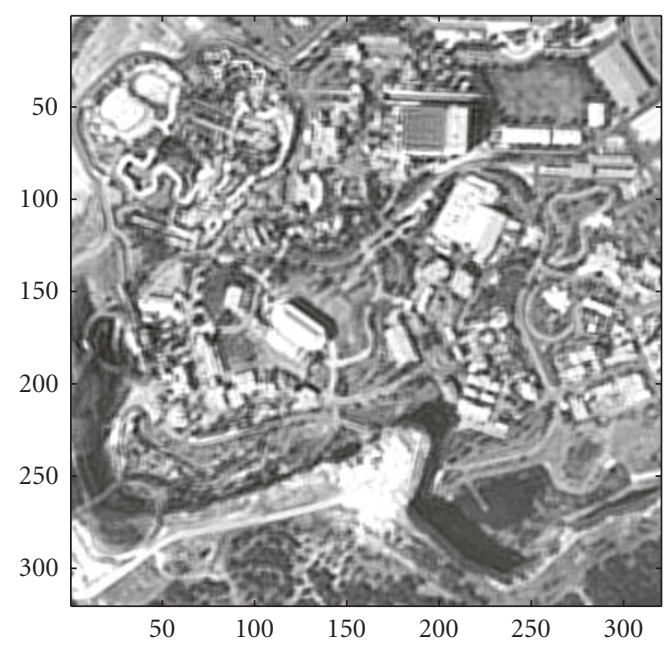

(d)

FIGURE 2: Simulated images: (a) true high-resolution image; (b) simulated frame-one low-resolution image; (c) observed frame-one lowresolution image with $\sigma_{n}^{2}=4$ and $\sigma_{b}^{2}=400$; (d) restored frame-one using the MAP SR-NUC algorithm for $P=30$ frames.

Substituting (14), (4), and (3) into (12) and removing scalars that are not functions of $\mathbf{z}$ or $\mathbf{b}$, we obtain the final cost function for simultaneous SR and NUC. This is given by

$$
\begin{aligned}
L(\mathbf{z}, \mathbf{b})= & \frac{1}{2 \sigma_{n}^{2}} \sum_{k=1}^{P}\left(\mathbf{y}_{k}-\mathbf{W}_{k} \mathbf{z}-\mathbf{b}\right)^{T}\left(\mathbf{y}_{k}-\mathbf{W}_{k} \mathbf{z}-\mathbf{b}\right) \\
& +\frac{1}{2} \mathbf{z}^{T} C_{z}^{-1} \mathbf{z}+\frac{1}{2 \sigma_{b}^{2}} \mathbf{b}^{T} \mathbf{b} .
\end{aligned}
$$

The cost function in (15) balances three terms. The first term on the right-hand side is minimized when a candidate $\mathbf{z}$, projected through the observation model, matches the observed data in each frame. The second term is minimized with a smooth HR image $\mathbf{z}$, and the third term is minimized when the individual biases are near zero. The variances $\sigma_{n}^{2}$, $\sigma_{z}^{2}$, and $\sigma_{b}^{2}$ control the relative weights of these three terms, where the variance $\sigma_{z}^{2}$ is contained in the covariance matrix
$C_{z}$ as shown by (4) and (5). It should be noted that the cost function in (15) is essentially the same as that used in the regularized least-squares method in [23]. The difference is that here we allow the observation model matrix $\mathbf{W}_{k}$ to include PSF blurring and downsampling, making this more general and appropriate for SR.

Next we consider a technique for minimizing the cost function in (15). A closed-form solution can be derived in a fashion similar to that in [23]. However, because the matrix dimensions are so large and there is a need for a matrix inverse, such a closed-form solution is impractical for most applications. In [23], the closed-form solution was only applied to a pair of small frames in order to make the problem computationally feasible. In the section below, we derive a gradient descent procedure for minimizing (15). We believe that this makes the MAP SR-NUC algorithm practical for many applications. 


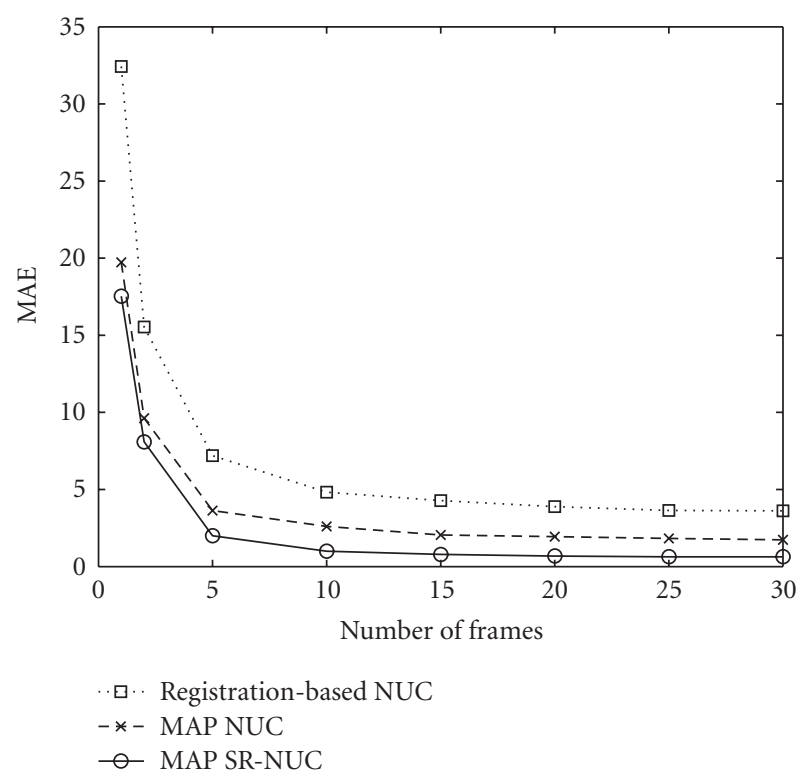

FIgURE 3: Mean absolute error for the estimated biases as a function of $P$ (the number of input frames).

\subsection{Gradient descent optimization}

The key to the optimization is to obtain the gradient of the cost in (15) with respect to the HR image $\mathbf{z}$ and the bias vector $\mathbf{b}$. It can be shown that the gradient of the cost function in (15) with respect to the HR image $\mathbf{z}$ is given by

$$
\nabla_{\mathbf{z}} L(\mathbf{z}, \mathbf{b})=\frac{1}{\sigma_{n}^{2}} \sum_{k=1}^{P} \mathbf{W}_{k}^{T}\left(\mathbf{W}_{k} \mathbf{z}+\mathbf{b}-\mathbf{y}_{k}\right)+C_{z}^{-1} \mathbf{z}
$$

Note that the term $C_{z}^{-1} \mathbf{z}$ can be expressed as

$$
C_{z}^{-1} \mathbf{z}=\left[\bar{z}_{1}, \bar{z}_{2}, \ldots, \bar{z}_{N}\right]^{T},
$$

where

$$
\bar{z}_{k}=\frac{1}{\sigma_{z}^{2}} \sum_{i=1}^{N} d_{i, k}\left(\sum_{j=1}^{N} d_{i, j} z_{j}\right) .
$$

The gradient of the cost function in (15) with respect to the bias vector $\mathbf{b}$ is given by

$$
\nabla_{\mathbf{b}} L(\mathbf{z}, \mathbf{b})=\frac{1}{\sigma_{n}^{2}} \sum_{k=1}^{P}\left(\mathbf{W}_{k} \mathbf{z}+\mathbf{b}-\mathbf{y}_{k}\right)+\frac{1}{\sigma_{b}^{2}} \mathbf{b} .
$$

We begin the gradient descent updates using an initial estimate of the HR image and bias vector. Here we lowpass filter and interpolate the first observed frame to obtain an initial HR image estimate $\mathbf{z}(0)$. The initial bias estimate is given by $\mathbf{b}(0)=\mathbf{0}$, where $\mathbf{0}$ is an $M \times 1$ vector of zeros. The gradient descent updates are computed as

$$
\begin{aligned}
\mathbf{z}(m+1) & =\mathbf{z}(m)-\varepsilon(m) \mathbf{g}_{\mathbf{z}}(m), \\
\mathbf{b}(m+1) & =\mathbf{b}(m)-\varepsilon(m) \mathbf{g}_{\mathbf{b}}(m),
\end{aligned}
$$

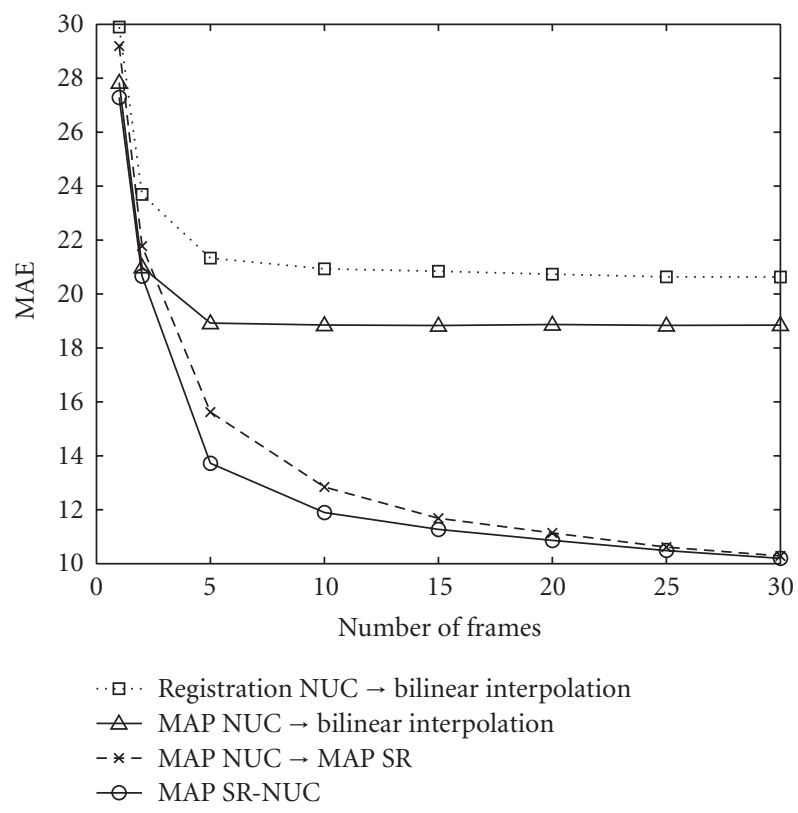

FIGURE 4: Mean absolute error for the HR image estimate as a function of $P$ (the number of input frames).

where $m=0,1,2, \ldots$ is the iteration number and

$$
\begin{aligned}
& \mathbf{g}_{\mathbf{z}}(m)=\left.\nabla_{\mathbf{z}} L(\mathbf{z}, \mathbf{b})\right|_{\mathbf{z}=\mathbf{z}(m), \mathbf{b}=\mathbf{b}(m)}, \\
& \mathbf{g}_{\mathbf{b}}(m)=\left.\nabla_{\mathbf{b}} L(\mathbf{z}, \mathbf{b})\right|_{\mathbf{z}=\mathbf{z}(m), \mathbf{b}=\mathbf{b}(m)} .
\end{aligned}
$$

Note that $\varepsilon(m)$ is the step size for iteration $m$. The optimum step size can be found by minimizing

$$
\begin{aligned}
& L(\mathbf{z}(m+1), \mathbf{b}(m+1)) \\
& \quad=L\left(\mathbf{z}(m)-\varepsilon(m) \mathbf{g}_{\mathbf{z}}(m), \mathbf{b}(m)-\varepsilon(m) \mathbf{g}_{\mathbf{b}}(m)\right)
\end{aligned}
$$

as a function of $\varepsilon(m)$. Taking the derivative of (22) with respect to $\varepsilon(m)$ and setting it to zero yields

$$
\begin{aligned}
\mathcal{E}(m)=\left(\frac{1}{\sigma_{n}^{2}} \sum_{k=1}^{P}\left(\mathbf{W}_{k} \mathbf{g}_{\mathbf{z}}(m)+\mathbf{g}_{\mathbf{b}}(m)\right)^{T}\left(\mathbf{W}_{k} \mathbf{z}(m)+\mathbf{b}(m)-\mathbf{y}_{k}\right)\right. \\
\left.\quad+\mathbf{g}_{\mathbf{z}}^{T}(m) C_{z}^{-1} \mathbf{z}(m)+\frac{1}{\sigma_{b}^{2}} \mathbf{g}_{\mathbf{b}}^{T}(m) \mathbf{b}(m)\right) / \\
\left(\frac{1}{\sigma_{n}^{2}} \sum_{k=1}^{P}\left(\mathbf{W}_{k} \mathbf{g}_{\mathbf{z}}(m)+\mathbf{g}_{\mathbf{b}}(m)\right)^{T}\left(\mathbf{W}_{k} \mathbf{g}_{\mathbf{z}}(m)+\mathbf{g}_{\mathbf{b}}(m)\right)\right. \\
\left.\quad+\mathbf{g}_{\mathbf{z}}^{T}(m) C_{z}^{-1} \mathbf{g}_{\mathbf{z}}(m)+\frac{1}{\sigma_{b}^{2}} \mathbf{g}_{\mathbf{b}}^{T}(m) \mathbf{g}_{\mathbf{b}}(m)\right) .
\end{aligned}
$$

We continue the iterations until the percentage change in cost falls below a pre-determined value (or a maximum number of iterations are reached).

\section{EXPERIMENTAL RESULTS}

In this section, we present a number of experimental results to demonstrate the efficacy of the proposed MAP estimator. 


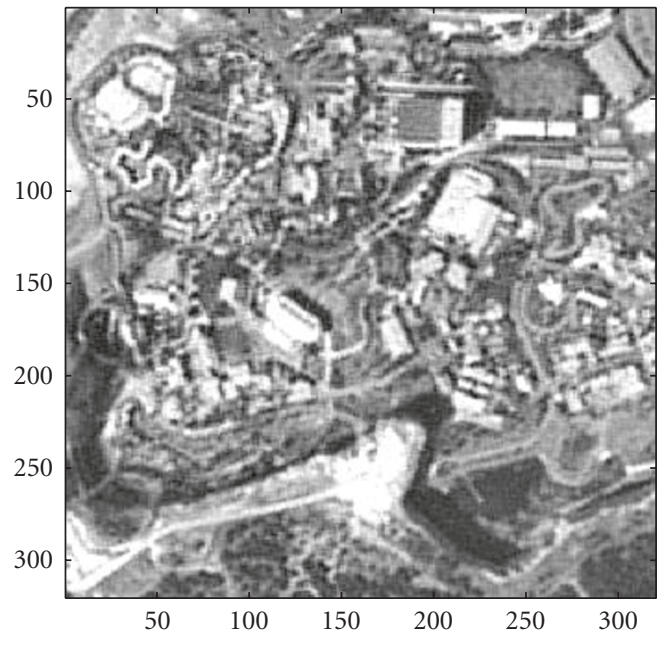

(a)

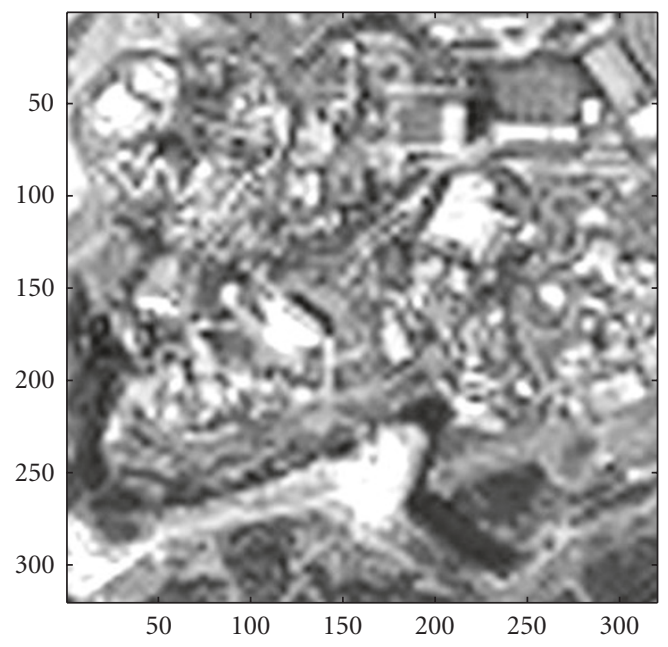

(c)

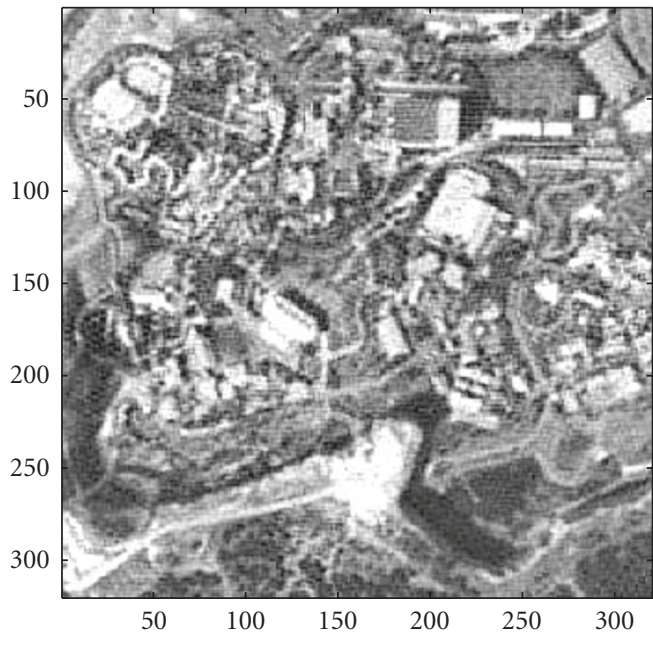

(b)

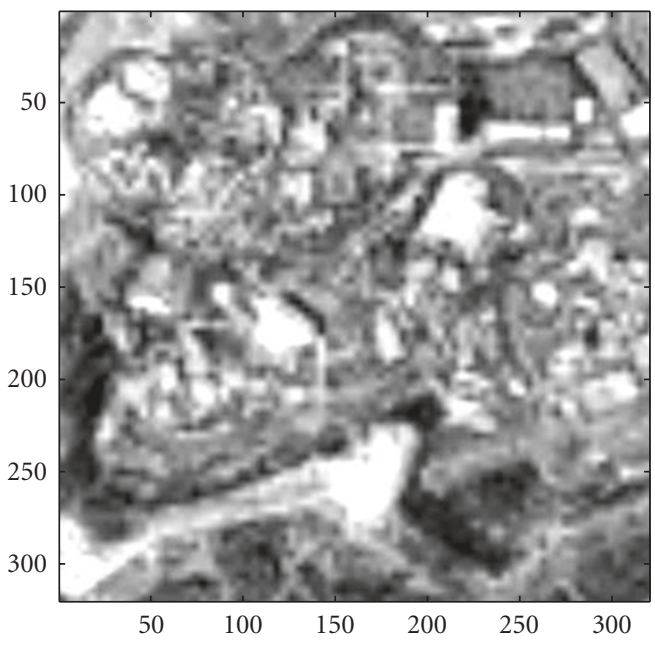

(d)

FIgURe 5: Simulated output HR image estimates for $P=5$ : (a) joint MAP SR-NUC; (b) MAP NUC followed by MAP SR; (c) MAP NUC followed by bilinear interpolation; (d) registration-based NUC followed by bilinear interpolation.

This first set of results is obtained using simulated imagery to allow for quantitative analysis. The second set uses real data from a forward-looking infrared (FLIR) imager to allow for qualitative analysis.

\subsection{Simulated data}

The original true HR image is shown in Figure 2(a). This is a single 8-bit grayscale aerial image to which we apply random translational motion using the model described in Section 2, downsample by $L_{x}=L_{y}=4$, introduce bias nonuniformity with variance $\sigma_{b}^{2}=40$, and add Gaussian noise with variance $\sigma_{n}^{2}=1$ to simulate a sequence of $30 \mathrm{LR}$ observed frames. The first simulated LR frame with $L_{x}=L_{y}=4$, slight translation and rotation, but no noise or nonuniformity, is shown in Figure 2(b). The first simulated observed frame with noise and nonuniformity applied is shown in
Figure 2(c). The output of the joint MAP SR-NUC algorithm is shown in Figure 2(d) for $P=30$ observed frames containing noise and nonuniformity. Here we used the exact motion parameters in the algorithm in order to assess the estimator independently from the motion estimation. An analysis of motion estimation in the presence of nonuniformity can be found in $[19,32,37]$. Note that for all the results shown here, we iterate the gradient descent algorithm until the cost decreases by less than $0.001 \%$ (typically 20-100 iterations).

The mean absolute error (MAE) for the bias estimates are shown in Figure 3 as a function of the number of input frames. We compare the joint MAP SR-NUC estimator with the MAP NUC algorithm (without SR, but equivalent to the MAP SR-NUC estimator with $L_{x}=L_{y}=1$ ) and the registration-based NUC proposed in [19]. Note that the joint MAP SR-NUC algorithm (with $L_{x}=L_{y}=4$ ) outperforms the MAP NUC algorithm $\left(L_{x}=L_{y}=1\right)$. Also note that both 

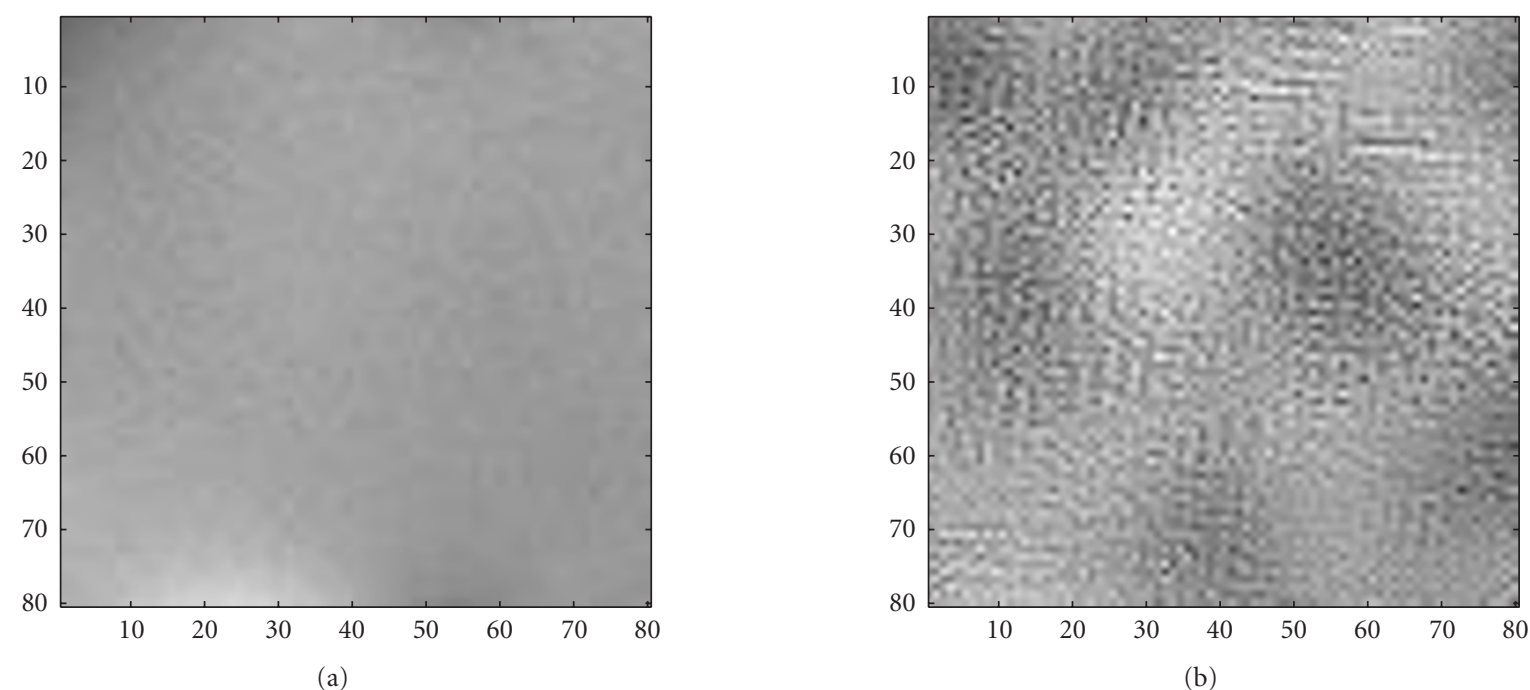

(b)

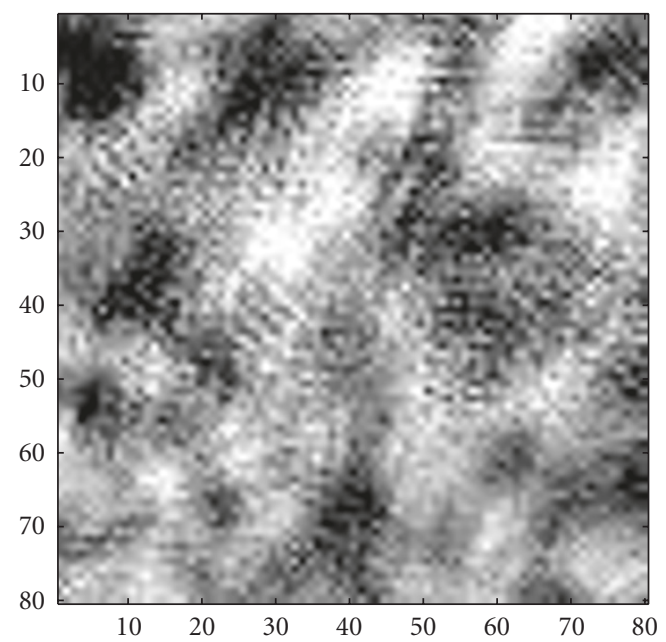

(c)

FIgURE 6: Bias error image for $P=30$ : (a) Joint MAP SR-NUC bias error image; (b) MAP NUC bias error image; (c) registration-based NUC bias error image.

MAP algorithms outperform the simple registration-based NUC method.

A plot of the MAE for the HR image estimates, versus the number of input frames, is shown in Figure 4. Here we compare the MAP SR-NUC algorithm to several two-step algorithms. Two of the benchmark approaches use the proposed MAP NUC $\left(L_{x}=L_{y}=1\right)$ algorithm to obtain bias estimates and these biases are used to correct the input frames. We consider processing these corrected frames using bilinear interpolation as one benchmark and using a MAP SR algorithm without NUC as the other. The pure SR algorithm is obtained using the MAP estimator presented here without the bias terms. This pure SR method is essentially the same as that in $[29,38]$. We also present MAEs for the registration-based NUC algorithm followed by bilinear interpolation. The error plot shows that for a small number of frames, the joint MAP SR-NUC estimator outperforms the two-step methods. For a larger number of frames, the error for the joint MAP SR-NUC and the independent MAP estimators is approximately the same. This is true even though Figure 3 shows that the bias estimates are more accurate using the joint estimator. This suggests that the MAP SR algorithm offers some robustness to the small nonuniformity errors when a larger number of frames are used (e.g., more than 30).

To allow for subjective performance evaluation of the algorithms, several output images are shown in Figure 5 for $P=5$. In particular, the output of the joint MAP SR-NUC algorithm is shown in Figure 5(a). The output of the MAP NUC followed by MAP SR is shown in Figure 5(b). The outputs of the MAP NUC followed by bilinear interpolation and registration-based NUC followed by bilinear interpolation are shown in Figures 5(c) and 5(d), respectively. Note that the adverse effects of nonuniformity errors are more 


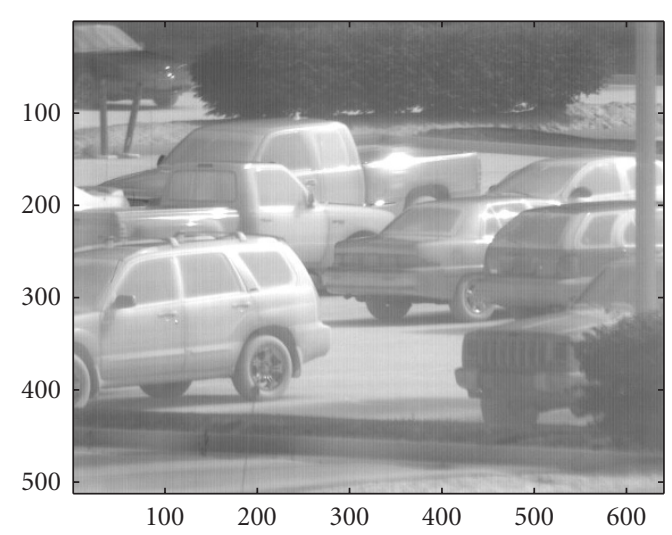

(a)

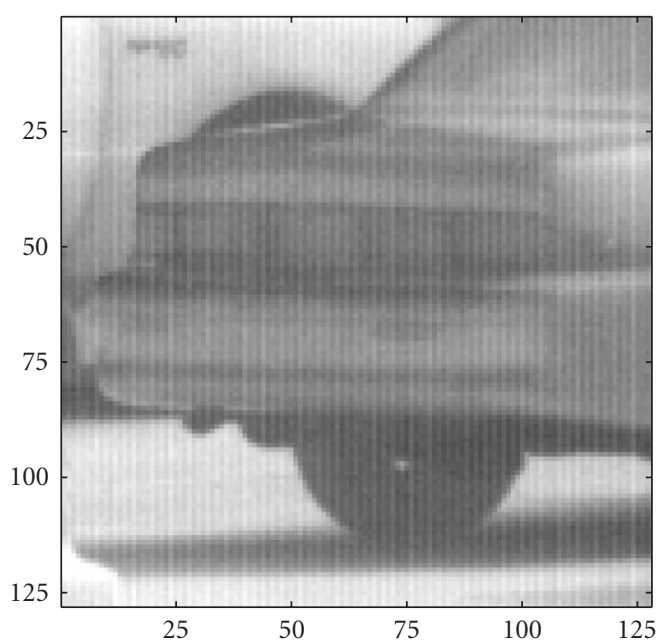

(b)

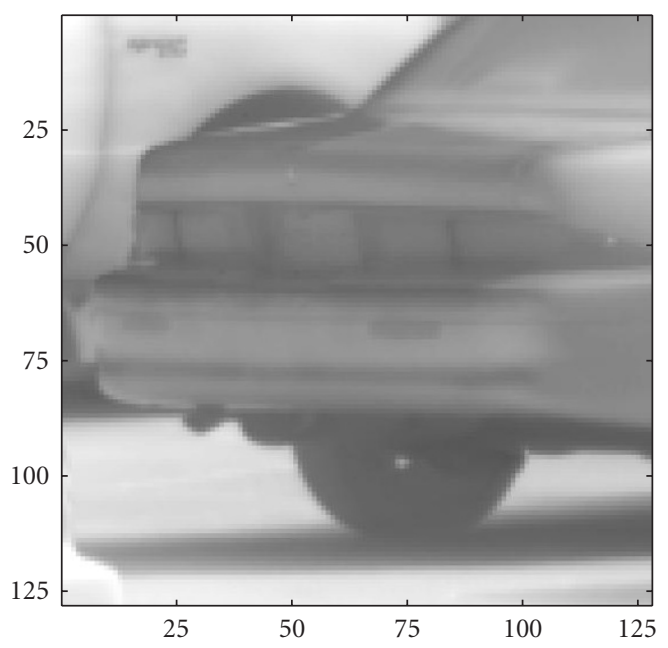

(d)

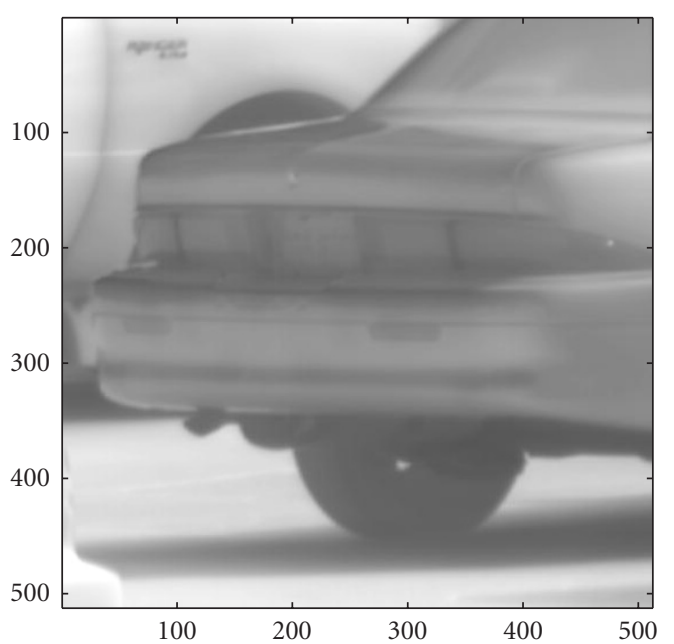

(c)

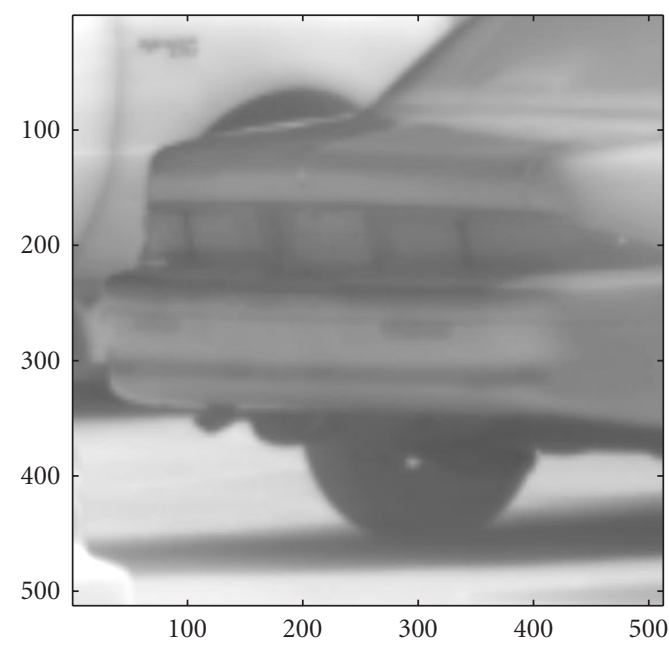

(e)

FIGURE 7: Simulated image results: (a) observed frame-one low-resolution image; (b) observed frame-one low-resolution image region of interest; (c) frame-one region of interest restored using the MAP SR-NUC algorithm for $P=20$ frames; (d) frame-one region of interest corrected with the MAP SR-NUC biases for $P=20$ frames; (e) low-resolution corrected region of interest followed by bilinear interpolation. 
evident in Figure 5(b) compared with those in Figure 5(a). The SR processed frames (Figures 5(a) and 5(b)) appear to have much greater details than those obtained with bilinear interpolation (Figures 5(c) and 5(d)), even with only five input frames. Additionally, the MAP NUC (Figure 5(c)) outperforms the registration-based NUC (Figure 5(d)).

To better illustrate the nature of the errors in the bias nonuniformity parameters, these errors are shown in Figure 6 as grayscale images. All of the bias error images are shown with the same colormap to allow for direct comparison. The middle grayscale value corresponds to no error. Bright pixels correspond to positive error and dark pixels correspond to negative error. The errors shown are for $P=30$ frames. The bias error for the joint MAP SR-NUC algorithm $\left(L_{x}=L_{y}=4\right)$ is shown in Figure 6(a). The error for the MAP NUC algorithm $\left(L_{x}=L_{y}=1\right)$ is shown in Figure 6(b). Finally, the bias error image for the registration-based method is shown in Figure 6(c). Note that with the joint MAP SRNUC algorithm, the bias errors have primarily low-frequency nature and their magnitudes are relatively small. The MAP NUC algorithm shows some high-frequency errors, possibly resulting from interpolation errors in the motion model. Such errors are reduced for the joint MAP SR-NUC method because the interpolation is done on the HR grid. The errors for the registration-based method include significant lowand high-frequency components.

\subsection{Infrared video}

In this section, we present the results obtained by applying the proposed algorithms to a real FLIR video sequence created by panning the camera. The FLIR imager contains a $640 \times 512$ infrared FPA produced by L-3 Communications Cincinnati Electronics. The FPA is composed of Indium-Antimonide ( $\mathrm{InSb}$ ) detectors with a wavelength spectral response of $3 \mu \mathrm{m}-5 \mu \mathrm{m}$ and it produces 14-bit data. The individual detectors are set on a $0.028 \mathrm{~mm}$ pitch, yielding a sampling frequency of 35.7 cycles $/ \mathrm{mm}$. The system is equipped with an $f / 4$ lens, yielding a cutoff frequency of $62.5 \mathrm{cycles} / \mathrm{mm}$ (undersampled by a factor of $3.5 \times$ ).

The full first raw frame is shown in Figure 7(a) and a center $128 \times 128$ region of interest is shown in Figure $7(\mathrm{~b})$. The output of the joint MAP SR-NUC algorithm for $L_{x}=L_{y}=4$ and $P=20$ frames is shown in Figure $7(\mathrm{c})$. Here we use $\sigma_{n}=5$, the typical level of temporal noise; $\sigma_{z}=300$, the standard deviation of the first observed LR frame; and $\sigma_{b}=100$, the standard deviation of the biases from a prior factory correction. We have observed that the MAP algorithm is not highly sensitive to these parameters and their relative values are all that impact the result. Here the motion parameters are estimated from the observed imagery using the registration technique detailed in $[38,39]$ with a lowpass prefilter to reduce the effects of the nonuniformity on the registration accuracy $[19,32,37]$.

The first LR frame corrected with the estimated biases is shown in Figure 7(d). The first LR frame corrected using the estimated bias followed by bilinear interpolation is shown in Figure $7(\mathrm{e})$. Note that the MAP SR-NUC image provides more details, including sufficient details to read the lettering on the side of the truck, than the image obtained using bilinear interpolation.

\section{CONCLUSIONS}

In this paper, we have developed a MAP estimation framework to jointly estimate an SR image and bias nonuniformity parameters from a sequence of observed frames. We use Gaussian priors for the HR image, biases, and noise. We employ a gradient descent optimization and estimate the motion parameters prior to the MAP algorithm. Here we estimate translation and rotation parameters using the method described in $[38,39]$.

We have demonstrated that superior results are possible with the joint method compared with comparable processing using independent NUC and SR. The bias errors were consistently lower for the joint MAP estimator with any number of input frames tested. The HR image errors were lower in our simulated image results using the joint MAP estimator when fewer than 30 frames were used. Our results suggest that a synergy exists between the SR and NUC estimation algorithms. In particular, the interpolation used for NUC is enhanced by the SR and the SR is enhanced by the NUC. The proposed MAP algorithm can be applied with or without SR, depending on the application and computational resources available. Even without SR, we believe that the proposed algorithm represents a novel and promising scene-based NUC technique. We are currently exploring nonuniformity models with gains and biases, more sophisticated prior models, alternative optimization strategies to enhance performance, and real-time implementation architectures based on this algorithm.

\section{REFERENCES}

[1] A. F. Milton, F. R. Barone, and M. R. Kruer, "Influence of nonuniformity on infrared focal plane array performance," Optical Engineering, vol. 24, no. 5, pp. 855-862, 1985.

[2] W. Gross, T. Hierl, and M. Schultz, "Correctability and longterm stability of infrared focal plane arrays," Optical Engineering, vol. 38, no. 5, pp. 862-869, 1999.

[3] D. L. Perry and E. L. Dereniak, "Linear theory of nouniformity correction in infrared staring sensors," Optical Engineering, vol. 32, no. 8, pp. 1854-1859, 1993.

[4] M. D. Nelson, J. F. Johnson, and T. S. Lomheim, "General noise processes in hybrid infrared focal plane arrays," Optical Engineering, vol. 30, no. 11, pp. 1682-1700, 1991.

[5] A. El Gamal and H. Eltoukhy, "CMOS image sensors," IEEE Circuits and Devices Magazine, vol. 21, no. 3, pp. 6-20, 2005.

[6] P. M. Narendra and N. A. Foss, "Shutterless fixed pattern noise correction for infrared imaging arrays," in Technical Issues in Focal Plane Development, vol. 282 of Proceedings of SPIE, pp. 44-51, Washington, DC, USA, April 1981.

[7] J. G. Harris, "Continuous-time calibration of VLSI sensors for gain and offset variations," in Smart Focal Plane Arrays and Focal Plane Array Testing, M. Wigdor and M. A. Massie, Eds., vol. 2474 of Proceedings of SPIE, pp. 23-33, Orlando, Fla, USA, April 1995.

[8] J. G. Harris and Y.-M. Chiang, "Nonuniformity correction using the constant-statistics constraint: analog and digital 
implementations," in Infrared Technology and Applications XXIII, B. F. Andresen and M. Strojnik, Eds., vol. 3061 of Proceedings of SPIE, pp. 895-905, Orlando, Fla, USA, April 1997.

[9] Y.-M. Chiang and J. G. Harris, "An analog integrated circuit for continuous-time gain and offset calibration of sensor arrays," Analog Integrated Circuits and Signal Processing, vol. 12, no. 3, pp. 231-238, 1997.

[10] D. A. Scribner, K. A. Sarkady, J. T. Caulfield, et al., "Nonuniformity correction for staring IR focal plane arrays using scenebased techniques," in Infrared Detectors and Focal Plane Arrays, E. L. Dereniak and R. E. Sampson, Eds., vol. 1308 of Proceedings of SPIE, pp. 224-233, Orlando, Fla, USA, April 1990.

[11] D. A. Scribner, K. A. Sarkady, M. R. Kruer, J. T. Caulfield, J. D. Hunt, and C. Herman, "Adaptive nonuniformity correction for IR focal-plane arrays using neural networks," in Infrared Sensors: Detectors, Electronics, and Signal Processing, T. S. Jayadev, Ed., vol. 1541 of Proceedings of SPIE, pp. 100-109, San Diego, Calif, USA, July 1991.

[12] D. A. Scribner, K. A. Sarkady, M. R. Kruer, et al., "Adaptive retina-like preprocessing for imaging detector arrays," in Proceedings of IEEE International Conference on Neural Networks, vol. 3, pp. 1955-1960, San Francisco, Calif, USA, March-April 1993.

[13] B. Narayanan, R. C. Hardie, and R. A. Muse, "Scene-based nonuniformity correction technique that exploits knowledge of the focal-plane array readout architecture," Applied Optics, vol. 44, no. 17, pp. 3482-3491, 2005.

[14] M. M. Hayat, S. N. Torres, E. E. Armstrong, S. C. Cain, and B. Yasuda, "Statistical algorithm for nonuniformity correction in focal-plane arrays," Applied Optics, vol. 38, no. 5, pp. 772-780, 1999.

[15] S. N. Torres and M. M. Hayat, "Kalman filtering for adaptive nonuniformity correction in infrared focal-plane arrays," Journal of the Optical Society of America A, vol. 20, no. 3, pp. 470480, 2003.

[16] R. C. Hardie and M. M. Hayat, "A nonlinear-filter based approach to detector nonuniformity correction," in Proceedings of IEEE-EURASIP Workshop on Nonlinear Signal and Image Processing, pp. 66-85, Baltimore, Md, USA, June 2001.

[17] W. F. O'Neil, "Dithered scan detector compensation," in Proceedings of the Infrared Information Symposium (IRIS) Specialty Group on Passive Sensors, Ann Arbor, Mich, USA, 1993.

[18] W. F. O’Neil, "Experimental verification of dither scan nonuniformity correction," in Proceedings of the Infrared Information Symposium (IRIS) Specialty Group on Passive Sensors, vol. 1, pp. 329-339, Monterey, Calif, USA, 1997.

[19] R. C. Hardie, M. M. Hayat, E. E. Armstrong, and B. Yasuda, "Scene-based nonuniformity correction with video sequences and registration," Applied Optics, vol. 39, no. 8, pp. 1241-1250, 2000.

[20] B. M. Ratliff, M. M. Hayat, and R. C. Hardie, "An algebraic algorithm for nonuniformity correction in focal-plane arrays," Journal of the Optical Society of America A, vol. 19, no. 9, pp. 1737-1747, 2002.

[21] B. M. Ratliff, M. M. Hayat, and J. S. Tyo, "Radiometrically accurate scene-based nonuniformity correction for array sensors," Journal of the Optical Society of America A, vol. 20, no. 10, pp. 1890-1899, 2003.

[22] B. M. Ratliff, M. M. Hayat, and J. S. Tyo, "Generalized algebraic scene-based nonuniformity correction algorithm," Journal of the Optical Society of America A, vol. 22, no. 2, pp. 239249, 2005.

[23] U. Sakoglu, R. C. Hardie, M. M. Hayat, B. M. Ratliff, and J. S. Tyo, "An algebraic restoration method for estimating fixed-pattern noise in infrared imagery from a video sequence," in Applications of Digital Image Processing XXVII, vol. 5558 of Proceedings of SPIE, pp. 69-79, Denver, Colo, USA, August 2004.

[24] S. C. Park, M. K. Park, and M. G. Kang, "Super-resolution image reconstruction: a technical overview," IEEE Signal Processing Magazine, vol. 20, no. 3, pp. 21-36, 2003.

[25] S. Borman, "Topics in multiframe superresolution restoration," Ph.D. dissertation, University of Notre Dame, Notre Dame, Ind, USA, April 2004.

[26] R. R. Schultz and R. L. Stevenson, "A Bayesian approach to image expansion for improved definition," IEEE Transactions on Image Processing, vol. 3, no. 3, pp. 233-242, 1994.

[27] P. Cheeseman, B. Kanefsky, R. Kraft, J. Stutz, and R. Hanson, "Super-resolved surface reconstruction from multiple images," Tech. Rep. FIA-94-12, NASA, Moffett Field, Calif, USA, December 1994.

[28] S. C. Cain, R. C. Hardie, and E. E. Armstrong, "Restoration of aliased video sequences via a maximum-likelihood approach," in Proceedings of National Infrared Information Symposium (IRIS) on Passive Sensors, pp. 230-251, Monterey, Calif, USA, March 1996.

[29] R. C. Hardie, K. J. Barnard, and E. E. Armstrong, "Joint MAP registration and high-resolution image estimation using a sequence of undersampled images," IEEE Transactions on Image Processing, vol. 6, no. 12, pp. 1621-1633, 1997.

[30] C. A. Segall, A. K. Katsaggelos, R. Molina, and J. Mateos, "Bayesian resolution enhancement of compressed video," IEEE Transactions on Image Processing, vol. 13, no. 7, pp. 898-910, 2004.

[31] E. E. Armstrong, M. M. Hayat, R. C. Hardie, S. N. Torres, and B. J. Yasuda, "Nonuniformity correction for improved registration and high-resolution image reconstruction in IR imagery," in Applications of Digital Image Processing XXII, A. G. Tescher, Ed., vol. 3808 of Proceedings of SPIE, pp. 150-161, Denver, Colo, USA, July 1999.

[32] E. E. Armstrong, M. M. Hayat, R. C. Hardie, S. N. Torres, and B. Yasuda, "The advantage of non-uniformity correction preprocessing on infrared image registration," in Application of Digital Image Processing XXII, vol. 3808 of Proceedings of SPIE, Denver, Colo, USA, July 1999.

[33] S. Cain, E. E. Armstrong, and B. Yasuda, "Joint estimation of image, shifts, and nonuniformities from IR images," in Infrared Information Symposium (IRIS) on Passive Sensors, Infrared Information Analysis Center, ERIM International, Ann Arbor, Mich, USA, 1997.

[34] S. Geman and D. Geman, "Stochastic relaxation, Gibbs distributions, and the Bayesian restoration of images," IEEE Transactions on Pattern Analysis and Machine Intelligence, vol. 6, no. 6, pp. 721-741, 1984.

[35] J. Besag, "Spatial interaction and the statistical analysis of lattice systems," Journal of the Royal Statistical Society B, vol. 36, no. 2, pp. 192-236, 1974.

[36] H. Derin and E. Elliott, "Modeling and segmentation of noisy and textured images using Gibbs random fields," IEEE Transactions on Pattern Analysis and Machine Intelligence, vol. 9, no. 1, pp. 39-55, 1987.

[37] S. C. Cain, M. M. Hayat, and E. E. Armstrong, "Projectionbased image registration in the presence of fixed-pattern noise," IEEE Transactions on Image Processing, vol. 10, no. 12, pp. 1860-1872, 2001.

[38] R. C. Hardie, K. J. Barnard, J. G. Bognar, E. E. Armstrong, and E. A. Watson, "High-resolution image reconstruction from a sequence of rotated and translated frames and its application 
to an infrared imaging system," Optical Engineering, vol. 37, no. 1, pp. 247-260, 1998.

[39] M. Irani and S. Peleg, "Improving resolution by image registration," CVGIP: Graphical Models and Image Processing, vol. 53, no. 3, pp. 231-239, 1991.

Russell C. Hardie graduated (magna cum laude) from Loyola College in Maryland in 1988 with the B.S. degree in engineering science. He obtained his M.S. and Ph.D. degrees in electrical engineering from the University of Delaware in 1990 and 1992, respectively. He served as a Senior Scientist at Earth Satellite Corporation in Maryland prior to his appointment at the University of Dayton in 1993. He is currently a Full

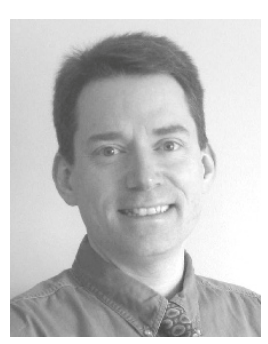
Professor in the Department of Electrical and Computer Engineering and holds a joint appointment with the Electro-Optics Program. Along with several collaborators, he received the Rudolf Kingslake Medal and Prize from SPIE in 1998 for work on multiframe image resolution enhancement algorithms. He recently received the University of Dayton's Top University-Wide Teaching Award, the 2006 Alumni Award in Teaching. In 1999, he received the School of Engineering Award of Excellence in Teaching at the University of Dayton and was the recipient of the first annual Professor of the Year Award in 2002 from the Student Chapter of the IEEE at the University of Dayton. His research interests include a wide variety of topics in the area of digital signal and image processing. His research work has focused on image enhancement and restoration, pattern recognition, and medical image processing.

Douglas R. Droege received both the B.S. degree in electrical engineering and the B.S. degree in computer science from the University of Dayton in 1999. In 2004, he obtained his M.S. degree in electrical engineering from the University of Dayton. He plans to graduate from the University of Dayton in 2008 with the Ph.D. degree in electrical engineering. He has spent seven years at L-

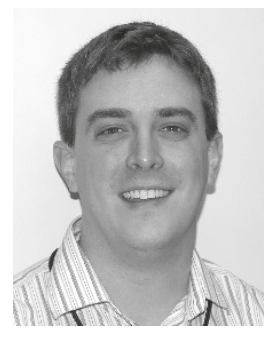
3 Communications Cincinnati Electronics developing infrared video signal processing algorithms and implementing them in real-time digital hardware. His research interests include image enhancement, detector nonuniformity correction, image stabilization, and superresolution. 